Revista de Dialectología y Tradiciones Populares, vol. LXXII, n. ${ }^{\circ}$ 1, pp. 23-28, enero-junio 2017 , ISSN: 0034-7981, eISSN: 1988-8457, doi: 10.3989/rdtp.2017.01.001.02

\title{
Interpretando la emigración de los jóvenes españoles en un contexto de crisis económica
}

\author{
Interpreting the Emigration of Young Spanish Adults \\ in a Context of Economic Crisis
}

\author{
Almudena Moreno Mínguez ${ }^{1}$ \\ Universidad de Valladolid
}

\section{RESUMEN}

Este artículo trata sobre los efectos de la crisis económica en la movilidad internacional de los jóvenes españoles desde una perspectiva crítica. Las dificultades para encontrar empleo derivadas de la crisis económica han propiciado un cambio sin precedentes en la movilidad internacional de los jóvenes españoles. Los resultados recogidos en este artículo apuntan a un relativo declive de la población joven debido a la reducción de la fecundidad y a la creciente movilidad de los jóvenes, hecho que por un lado ha generado un gran ruido mediático y por otro puede tener consecuencias futuras para la sostenibilidad del Estado de bienestar en España.

Palabras clave: Movilidad internacional; Jóvenes; Crisis económica; Declive poblacional.

\section{SUMMARY}

This article discusses the effects of the economic crisis on the international mobility of young Spaniards from a critical perspective. The difficulties in finding employment resulting from the economic crisis have changed the international mobility of young people. The results presented in this paper point to a relative decline in the young population due to reduced fertility and increased youth mobility. On the one hand, this new trend has generated a relative media distortion. On the other hand, it could have future implications for the sustainability of the Spanish welfare state.

Key words: International Mobility; Youth; Economic Crisis; Population Decline.

\footnotetext{
${ }^{1}$ Correo electrónico almudena@soc.uva.es. ORCID ID: http://orcid.org/0000-0002-1839-0508.
} 


\section{INTRODUCCIÓN}

Quizás uno de los temas más tratados en la literatura y en los medios de comunicación haya sido el efecto de la crisis económica en la movilidad creciente de los jóvenes españoles (López Sala y Oso 2015). A este respecto se ha destacado el desempleo y la emigración de los jóvenes como dos elementos interdependientes resultantes de la crisis económica. Sin embargo estas versiones mediáticas no siempre reflejan el significado real que describen los datos sobre el desempleo y la emigración juvenil (González Enríquez 2012). Esta sobredimensionada realidad mediática se explicaría en el contexto de los cambios que ha experimentado la condición juvenil en las últimas décadas en España y del declive de la población joven. Estas transformaciones se evidencian en la prolongación de las transiciones formativo-laborales, residenciales y familiares, así como en los estilos de vida; en definitiva, en las decisiones que adoptan los jóvenes para adaptarse a un entorno cambiante. Estas decisiones responden, en parte, a factores estructurales como los demográficos, los económicos y los políticos, pero también a factores generacionales del curso de la vida (Arnett 2004; Lecardi y Ruspini 2016; Moreno 2016; Brückner y Mayer 2005).

Son múltiples los motivos que mueven a los jóvenes a emigrar de su región o país de origen. Entre ellos están los económicos para mejorar la formación y encontrar un empleo y otros que tienen que ver con motivos culturales y personales propios del ciclo vital en el que viven (Massey et al. 1993; Punch 2007). En el caso español, la emigración juvenil de las últimas décadas previas a la crisis económica ha sido relativamente reducida debido a la escasa movilidad que caracterizaba a la sociedad española desde la transición democrática. Sin embargo, a raíz de la crisis, aumenta el número de jóvenes que deciden marcharse al extranjero, lo que despierta un inusitado interés mediático. Los motivos son básicamente los señalados en la literatura internacional (McKenzie 2008; Yaqub 2009) y que se resumen básicamente en económicos (encontrar una oportunidad laboral) y poder realizar la transición a la vida adulta para adquirir cierta independencia residencial y económica (INJUVE 2014).

El objetivo que nos proponemos en este artículo es por tanto reflexionar críticamente sobre el fenómeno migratorio de los jóvenes, con el fin de poder elaborar un relato interpretativo desde una perspectiva holística que aporte significados al mediático fenómeno de la movilidad internacional de los jóvenes.

\section{LA EMIGRACIÓN JUVENIL EXPLICADA DESDE LA DEMOGRAFÍA Y LA PRECARIEDAD ECONÓMICA Y LABORAL}

La interpretación de la movilidad internacional de los jóvenes no puede entenderse sin preguntarse sobre las consecuencias poblacionales y socio-económicas que ha tenido la segunda transición demográfica en el colectivo de los jóvenes en España (Lesthaeghe 2010). Es bien conocido por los demógrafos los efectos del envejecimiento de la población en las transiciones juveniles y en las economías nacionales como consecuencia del declive de la fecundidad (Billari y Kohler 2004; Aassve et al. 2002). En España el declive poblacional se inicia a partir de 1975, momento en el que se empieza a registrar un descenso continuado en el número de nacimientos (Livi Bacci 1986; 
Muñoz 1995). La consecuencia inmediata de esta dinámica es la reducción de las cohortes de población, lo que se reflejará en la pirámide de población y más concretamente en la reducción de la población infantil y juvenil. Esto supone un nuevo escenario poblacional en el que los jóvenes serán cada vez menos numerosos, mientras las cohortes de población más envejecidas incrementarán su peso poblacional. Este fenómeno no sólo tendrá efectos demográficos sino también económicos, sociales, políticos, culturales y mediáticos. La disminución progresiva de las cohortes de población joven no parece ser un hecho coyuntural sino estructural que se mantendrá en el tiempo, según las proyecciones del Instituto Nacional de Estadística. En definitiva, la progresiva reducción de las cohortes poblacionales de los jóvenes, resultado de la reducción de la fecundidad y en menor medida de la creciente movilidad geográfica a otros países, sobre todo en los últimos ocho años, ha propiciado cierto "temor a quedarnos sin jóvenes", dando lugar a un intenso debate mediático en torno a las consecuencias de la movilidad internacional de los jóvenes españoles, que no siempre se corresponden con el significado real del fenómeno migratorio (OCDE 2015).

A esta crisis demográfica se une la crisis económica y laboral cuya expresión máxima es el desempleo juvenil y la precariedad económica en la que viven los jóvenes. El incremento del desempleo juvenil, consecuencia de la crisis económica, con especial intensidad en España, ha propiciado un intenso debate académico e institucional sobre las consecuencias del desempleo en la población juvenil (Banco de España 2013; Dolado 2015). Un problema asociado con el desempleo estructural que sufre España es por un lado la reducida empleabilidad de los jóvenes descualificados y sin formación y por otro lado el desaprovechamiento del talento y de la inversión en capital humano (Moreno 2015). Estos datos apuntan a que el sistema productivo español tiene problemas serios para absorber a trabajadores jóvenes formados y cualificados, lo que podría ser interpretado como una causa laboral y económica del crecimiento del flujo migratorio de los jóvenes españoles a partir del año 2008.

\section{EL RELATO INTERPRETATIVO DE LA EMIGRACIÓN JUVENIL A TRAVÉS DE LOS DATOS}

La pregunta que suscita el escenario demográfico y laboral definido previamente es en qué medida la movilidad juvenil se ha convertido en un fenómeno generalizado y qué consecuencias puede tener para la sociedad española. La reflexión que aporta este artículo parte de la idea de que la emigración juvenil en España es una respuesta individual a los efectos de la crisis económica en un contexto institucional y económico en el que los jóvenes han visto mermadas sus oportunidades de integrarse en el mercado laboral y de transitar hacia la independencia residencial y la formación de la familia. Sin embargo, se trata de un fenómeno en muchos casos sobredimensionado por los medios de comunicación y que se explica en parte como consecuencia de la escasa movilidad que ha caracterizado a la población española en las últimas décadas. Los estudios realizados han subrayado que la decisión de emigrar del lugar de origen se explican en función de la búsqueda de mejores oportunidades de formación y de empleo (INJUVE 2014; Cortés et al. 2015; González Enríquez 2012; González Ferrer 2013). 
Los datos disponibles para valorar el significado real de la emigración internacional juvenil son confusos debido a las limitaciones de las fuentes disponibles y a la complejidad de las dimensiones que implica (movilidad nacional, europea y trans-europea). En España contamos con tres fuentes estadísticas disponibles para el estudio de las migraciones que son: el Censo Electoral de Españoles Residentes en el Extranjero (CERA) que se utiliza como censo electoral, la Encuesta de Variaciones Residenciales (EVR) que elabora el INE, basado en las altas y bajas producidas en el Padrón, y el Padrón de Españoles Residentes en el Extranjero (PERE). El principal problema de estas fuentes es que la inscripción en las mismas es voluntaria, por lo que no recogen el flujo real de personas que se mueven sin inscribirse en estos registros. En base a estas limitaciones estadísticas es difícil conocer con rigor cuántos jóvenes españoles han emigrado durante la crisis, aunque estas fuentes sí que nos permiten al menos tener una aproximación al fenómeno. Según un estudio del INJUVE (2014), la cifra estimada de jóvenes emigrantes entre 2007 y 2013 sería de 341.000, número menor al que apuntan algunos estudios pero mayor que la cifra que aporta el PERE de 218.000. Si bien los datos disponibles no responden a la visión alarmista que transmiten muchos medios de comunicación, sí que reflejan un cambio de tendencia, ya que

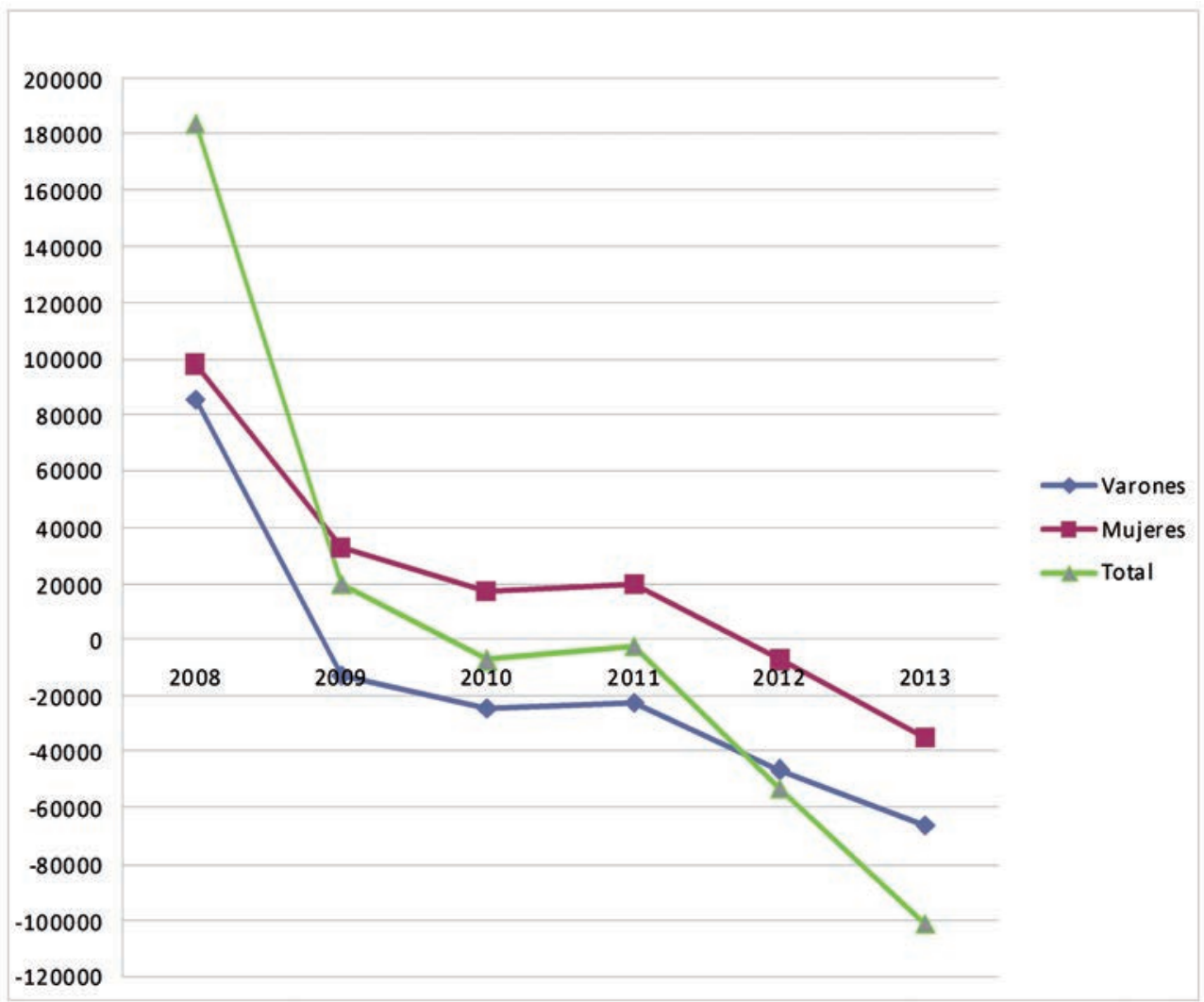

GRÁFICO 1.-Saldo migratorio al extranjero, jóvenes 15-34 años. Fuente: Elaboración propia a partir de datos del Instituto Nacional de Estadística (varios años). 
la sociedad española ha sido relativamente inmóvil (en términos de desplazamientos geográficos) desde mediados de los años 70, donde la resistencia de los españoles a desplazarse a otros países era una de las principales trabas para la internacionalización de nuestro mercado productivo. Sin embargo, a raíz de la crisis económica, algo cambia y la población española - y concretamente los jóvenes- empiezan a asumir la movilidad como un requisito imprescindible para adaptarse a un mundo global y conseguir nuevas oportunidades de empleo que no ofrece su entorno local.

Un indicador de interés para valorar el significado de la emigración juvenil es el saldo migratorio. El Gráfico 1 destaca el hecho de que a pesar del incremento de las salidas al exterior de jóvenes en los dos primeros años de referencia, entre 2008 y 2010 se produce una caída de más de 180.000 jóvenes, descenso que se prolongará hasta el año 2013. Estos datos son reveladores del cambio de tendencia que supone la irrupción de la crisis en la movilidad de los jóvenes.

Otro efecto mediático no contrastado sobre la emigración de los jóvenes se refiere a la supuesta "fuga de cerebros". Los datos disponibles en origen y destino no incluyen información desagregada sobre el nivel de cualificación de los jóvenes emigrados, por lo que el debate mediático sobre la "fuga de cerebros" es ambiguo y en cierta manera no está fundamentado empíricamente. A este respecto, González-Ferrer (2013) se refiere al término de "migración cualificada", que suele utilizarse para designar la migración de personas con educación superior completada y que resulta, por tanto, más adecuado para referirse a este fenómeno. La pregunta que se plantea es si la emigración juvenil continuará y si retornarán los jóvenes que emigraron durante la crisis. Según los cálculos de Gaule (2011), tan sólo retorna el 10\% de los científicos y académicos emigrados, lo cual no son buenas noticias.

\section{CONCLUSIÓN}

Quizás uno de los fenómenos más referenciados en los medios de comunicación en España haya sido los efectos que la crisis económica ha tenido en el desempleo y la emigración juvenil. Los datos recogidos en este capítulo apuntan al declive de las cohortes de población más jóvenes, lo que unido al elevado desempleo juvenil como un hecho estructural de nuestro mercado laboral, pero agravado por la crisis, ha supuesto un cambio de tendencia en la movilidad geográfica internacional de los jóvenes, que hasta el inicio de la crisis era relativamente reducida. Si bien se trata de un fenómeno complejo de difícil medición, los datos disponibles sobre la movilidad internacional de los jóvenes apuntan a un incremento relativo del número de jóvenes que han salido del país durante la crisis, lo que no se corresponde con la alarma social generada por los medios de comunicación. Si interpretamos en conjunto el declive poblacional del colectivo de jóvenes, derivado del declive de la fecundidad, con el cambio de tendencia experimentado en la movilidad de los jóvenes españoles, estamos ante un nuevo escenario en el que los jóvenes son cada vez menos en la pirámide de población, en la población activa y en los censos electorales, lo que puede tener consecuencias en el diseño de las políticas de juventud y en la sostenibilidad del Estado de bienestar español. En definitiva, estamos ante un fenómeno social complejo y diverso, cuyas consecuencias inmediatas y a medio y largo plazo requieren disponer de una información mucho más actualizada y de calidad para ser correctamente evaluado y poder diseñar políticas públicas acordes con ese nuevo escenario social. 


\section{BIBLIOGRAFÍA CITADA}

Aassve, Arnstein, F. C. Billari, S. Mazzuco y F. Onagro. 2002. "Leaving Home: A Comparative Analysis of ECHPdata". Journal of European Social Policy 12(4): 259-275.

Arnett, Jeffrey. 2004. Emerging Adulthood: The Winding Road from the Late Teens through the Twenties. Nueva York: Oxford University Press.

Banco de España. 2013. "Evolución del desajuste educativo entre la oferta y la demanda de trabajo en España”. Boletín Económico 6: 43-50.

Billari, Francisco y Hans-Peter Kohler. 2004. "Patterns of Low and Lowest-Low Fertility in Europe". Population Studies 58(2): 161-176.

Brückner, Hannah y Ulrich Mayer. 2005. "De-Standardization of the Life Course: What It Might Mean? And if It Means Anything, Whether It Actually Took Place?", en Ross Macmillan (ed.), The Structure of the Life Course: Standardized? Individualized? Differentiated?: 27-53. Ámsterdam: Elsevier.

Cortés, Almudena, Beatriz Moncó y Débora Betrisey. 2015. Movilidad transnacional de jóvenes españoles y latinoamerticanos: una comparación en contexto de crisis. Madrid: Centro Reina Sofía sobre Juventud y Adolescencia.

Dolado, Juan (ed.). 2015. No Country for Young People? Youth Labour Market Problems in Europe. Londres: CEPR Press.

Gaule, Patrick. 2011. Return Migration: Evidence from Academic Scientists. Mimeo Massachusetts, Cambridge: Institute of Technology.

González Enríquez, Carmen. 2012. La emigración desde España, una migración de retorno. Real Instituto Elcano. Disponible en: <http://www.realinstitutoelcano.org/wps/portal/rielcano/contenido?WCM_ GLOBAL_CONTEXT=/elcano/elcano_es/zonas_es/ari4-2012>. Fecha de acceso: 05 abr. 2017.

González-Ferrer, Amparo. 2013. "La nueva emigración española. Lo que sabemos y lo que no". Laboratorio de Alternativas 18: 1-19. Disponible en: <http://digital.csic.es/bitstream/10261/93167/ 1/La\%20nueva\%20emigraci\%C3\%B3n\%20espa\%C3\%B1ola.pdf>. Fecha de acceso: 05 abr. 2017.

Lecardi, Carmen y Elisabetta Ruspini. 2006. A New Youth? New People, Generations and Family Life. Aldershot: Ashgate.

Lesthaeghe, Ron. 2010. "The Unfolding Story of the Second Demographic Transition". Population and Development Review 36(2): 211-251.

Livi Bacci, Massimo. 1986. "Social-Group Forerunners of Fertility Control in Europe", en Ansley Johnson Coale y Sussan Cotts Watwins (eds.), The Decline of Fertility in Europe. Princeton: Princeton University Press.

López Sala, Anna y Laura Oso. 2015. "Inmigración en tiempos de crisis: dinámicas de movilidad emergentes y nuevos impactos sociales". Migraciones 37: 9-27.

Massey, Douglas, Joaquín Arango, Hugo Graeme, Ali Kouaqoui, Adela Pellegrino y Edward Taylor. 1993. "Theories of International Migration: A Review and Appraisal". Population and Development Review 19(3): 431-466.

McKenzie, David. 2007. "A Profile of the World's Young Developing Country Migrants". IZA Discussion Paper 2948. Bonn: Institute for the Study of Labour (IZA).

Moreno Mínguez, Almudena. 2015. "La empleabilidad de los jóvenes en España: explicando el elevado desempleo juvenil durante la recesión económica”. Revista Internacional de Investigación en Ciencias Sociales 11(1): 3-20.

Moreno Mínguez, Almudena. 2016. "Economic Crisis and the New Housing Transitions of Young People in Spain". International Journal of Housing Policy 16: 165-183.

Muñoz Pérez, Francisco. 1995. "Procreación y matrimonio en España (1970-1990)". Revista Internacional de Sociología 11: 197-238.

OCDE. 2015. International Migration Outlook, 2015. París: OCDE.

Punch, Samantha. 2007. "Migration Projects: Children on the Move for Work and Education". Artículo presentado a Independent Child Migrants: Policy Debates and Dilemmas organizado por el Development Research Centre on Migration, Globalisation and Poverty. 12-09-2007. Londres: UNICEF Innocenti Research Centre.

Yaqub. Shahin. 2009. "Independent Child Migrants in Developing Countries: Unexplored Links in Migration and Development". Discussion Paper, IWP-2009-01. Florencia: UNICEF Innocenti Research Centre. 